\title{
Urticaria and Angioedema - More than just Skin Deep!
}

\author{
Holtzhausen J \\ Allergy Diagnostic and Clinical Research Unit and Lung Clinical Research Unit, University of Cape Town Lung Institute, Cape Town
}

*Corresponding author, email: jeanette.holtzhausen@uct.ac.za

\begin{abstract}
Urticaria and angioedema are characterized by pruritic hives and sometimes swelling of deeper mucocutaneous layers. Urticaria is caused by release of histamine and other mediators from mast cells. A cut-off of six weeks distinguishes acute and chronic forms, as these seem to differ regarding etiological and response patterns. Angioedema may be histaminergic or due to factors involving the bradykinin pathway. Medications such as ACE-Inhibitors or conditions affecting the C1-esterase inhibitor enzyme are potential causes.
\end{abstract}

Acute urticaria may be related to triggers such as infections, medications or food allergy. When occurring with systemic symptoms and a temporal relationship to food or drug ingestion, IgE mediated allergy could be considered.

Chronic Inducible Urticaria (CInd) is suspected on history and may be confirmed by provocation testing where physical and other specific trigger factors elicit the wheals.

The term Chronic Spontaneous Urticaria (CSU) is used when symptoms persist for more than six weeks in the absence of inducible factors. A single cause is seldom found but a thorough history and clinical evaluation may elicit trigger factors and associated medical conditions such as auto-immune or thyroid disorders. Indiscriminate food allergy testing is unhelpful, but additive intolerance may be implicated in some cases. Modern non-sedating antihistamines are the mainstay of treatment, and omalizumab or immunosuppressives may be considered for refractory cases. Emotional support is needed as the condition is associated with psychological distress.

If there are unusual features, vasculitis, auto-inflammatory or haematological conditions should be considered and skin biopsy or detailed immunological work-up may be indicated.

Keywords: Angioedema, urticaria, chronic spontaneous, mast cells, pruritus, itch, histamine, hive, wheals, omalizumab

\section{Introduction}

Family practitioners frequently encounter patients presenting with urticaria and angioedema. Patients attribute the symptoms to allergy, but the answer is not usually straightforward. The clinician needs to think like a detective and use a detailed history and clinical evaluation to inform the choice of investigations. The purpose of this article is to give a broad overview and practical approach, and then touch on current guidelines and recent developments regarding management of Chronic Spontaneous Urticaria in particular.

\section{Pathophysiology and classification}

The Latin root of the word urticaria is urere, meaning 'to burn', as is evident in the family name of the Urticaceae stinging nettle plants. The classic 'wheal and flare' histamine response is illustrated by the discomfort experienced upon cutaneous contact with these plants. The polymorphic, raised and erythematous lesions of urticaria are due to vasodilation and increased vascular permeability, following release of mediators by cutaneous mast cells. ${ }^{1-4}$ Individual urticarial lesions usually resolve within 24 hours, without scarring, as the epidermis is not involved. ${ }^{5}$ The rash is intensely pruritic and tends to 'migrate' or become confluent.

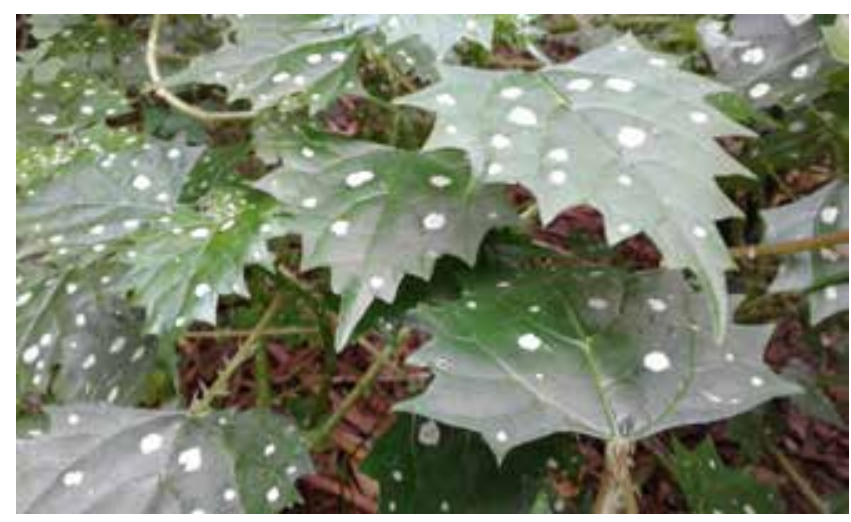

Urticaceae species stinging nettle plant 


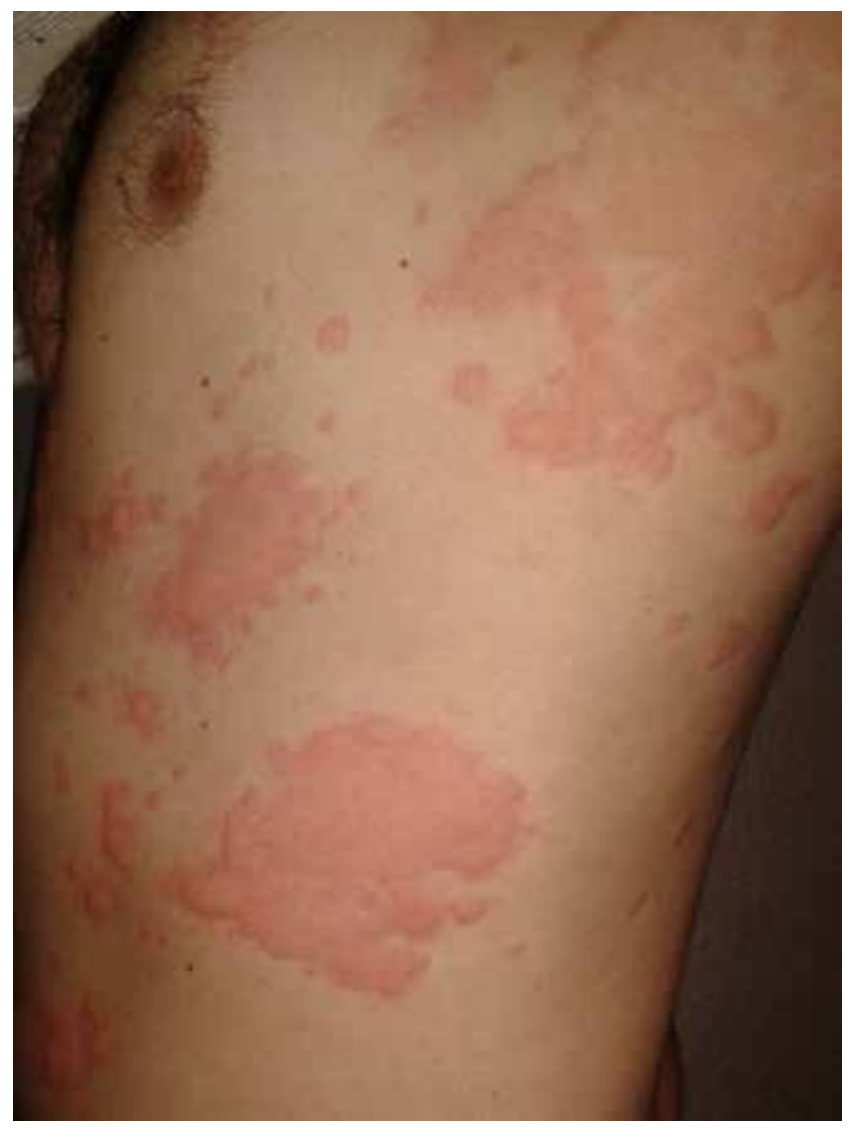

Typical urticarial skin rash

Angioedema occurs when deeper mucocutaneous layers are affected, temporarily disfiguring the face, eyelids and lips. Lesions are painful and can take up to three days to resolve. When occurring in isolation, the bradykinin pathway may be involved and the airway is more likely to be threatened. Angiotensin Converting Enzyme inhibitors are a common cause, even after longstanding use, and should be discontinued permanently. Hereditary or acquired deficiency, or functional impairment, of the $\mathrm{C} 1$ esterase enzyme should be also considered, especially if there is a family history of swelling, airway compromise or surgical complications indicating possible hereditary angioedema. Diagnostic tests include laboratory evaluation of C3, C4 and C1 esterase levels. Acquired C1 esterase deficiency may occasionally be linked to underlying malignant conditions.

When urticaria is the dominant symptom, or occurs together with angioedema, the process is usually mast cell mediated. Mast cells contain cytoplasmic granules holding pre-formed substances such as histamine and proteases, release of which causes intense itching, burning, vasodilatation and recruitment of other inflammatory mediators. Mast cell activation is complex and initiated by many signals, including lgE mediated allergy, auto-antibodies, direct chemical irritation and physical triggers through specific mast cell surface receptors. ${ }^{10}$

Acute urticaria of less than 6 weeks' duration may present with other symptoms, e.g. oral itching, wheezing, gastrointestinal symptoms or cardiovascular compromise due to IgE mediated allergy. ${ }^{5}$ The cause may be elicited from history and investigations, e.g. food, drug, contact or insect allergy or recent infections. Skin prick or ImmunoCAP specific IgE tests for specific allergens may be helpful. An example is recent onset of crustacean shellfish allergy in a patient who reports oral itching, wheeze and urticarial hives developing within hours after eating shrimp in a restaurant.

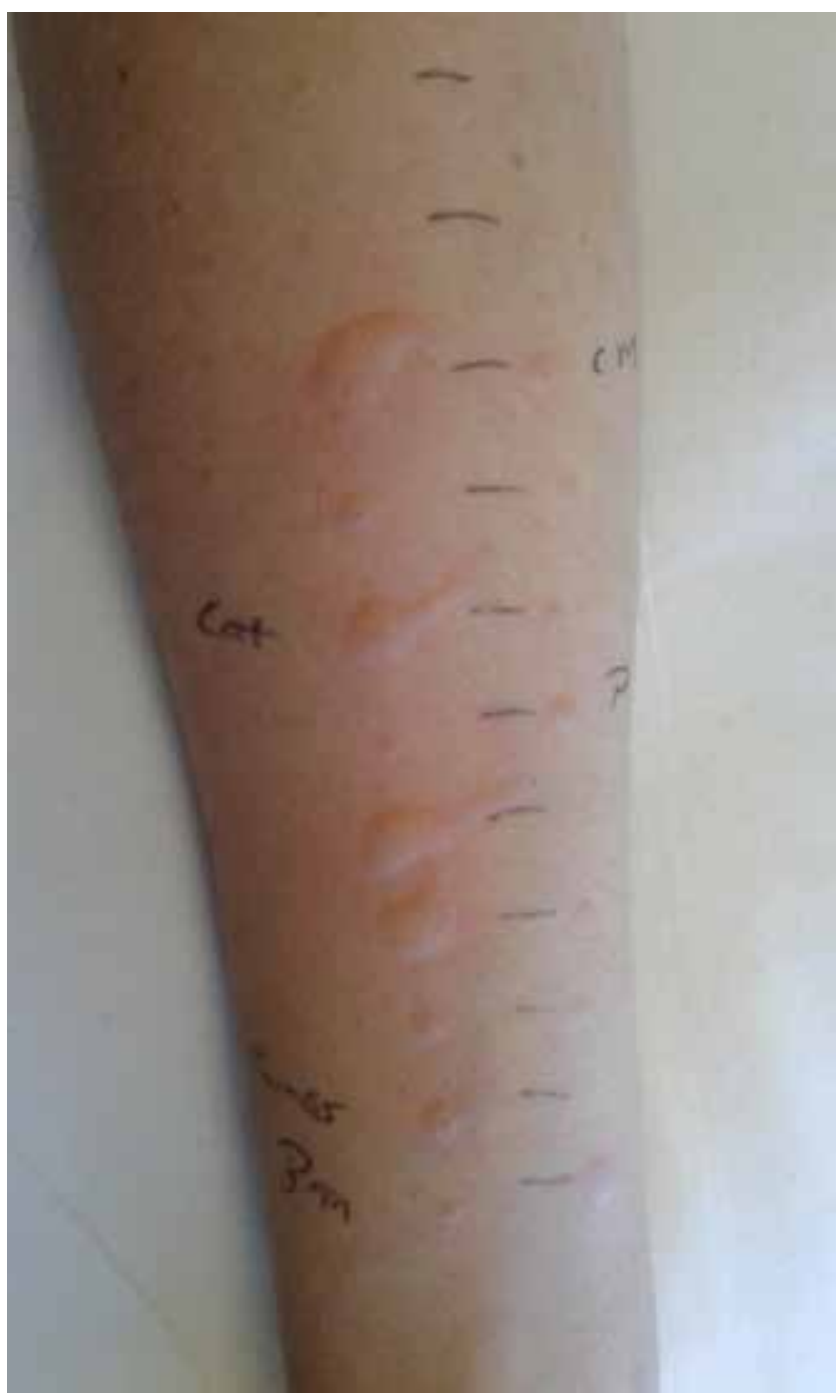

Skin prick testing for IgE mediated allergy

Inducible urticarias are diagnosed when wheals occur in response to specific trigger factors. These include physical factors, e.g. dermatographism, pressure, temperature, sunlight, vibration and others such as cholinergic, aquagenic and contact urticaria. Diagnosis is based on history and sometimes provocation testing, for example the ice-cube test for cold urticaria. ${ }^{5}$

Chronic Spontaneous Urticaria (CSU) is a heterogeneous disorder characterized by the presence of pruritic hives, with or without angioedema, for longer than six weeks. ${ }^{2}$ The term 'Chronic Idiopathic Urticaria' is a synonym. It is incompletely understood and remains a management challenge, as not all patients respond to treatment. ${ }^{1,2} \mathrm{~A}$ single cause is seldom found and reflex requests for inappropriate food allergy testing panels should be discouraged as it is expensive and results are unlikely to be clinically significant. Associations do, however, exist with 


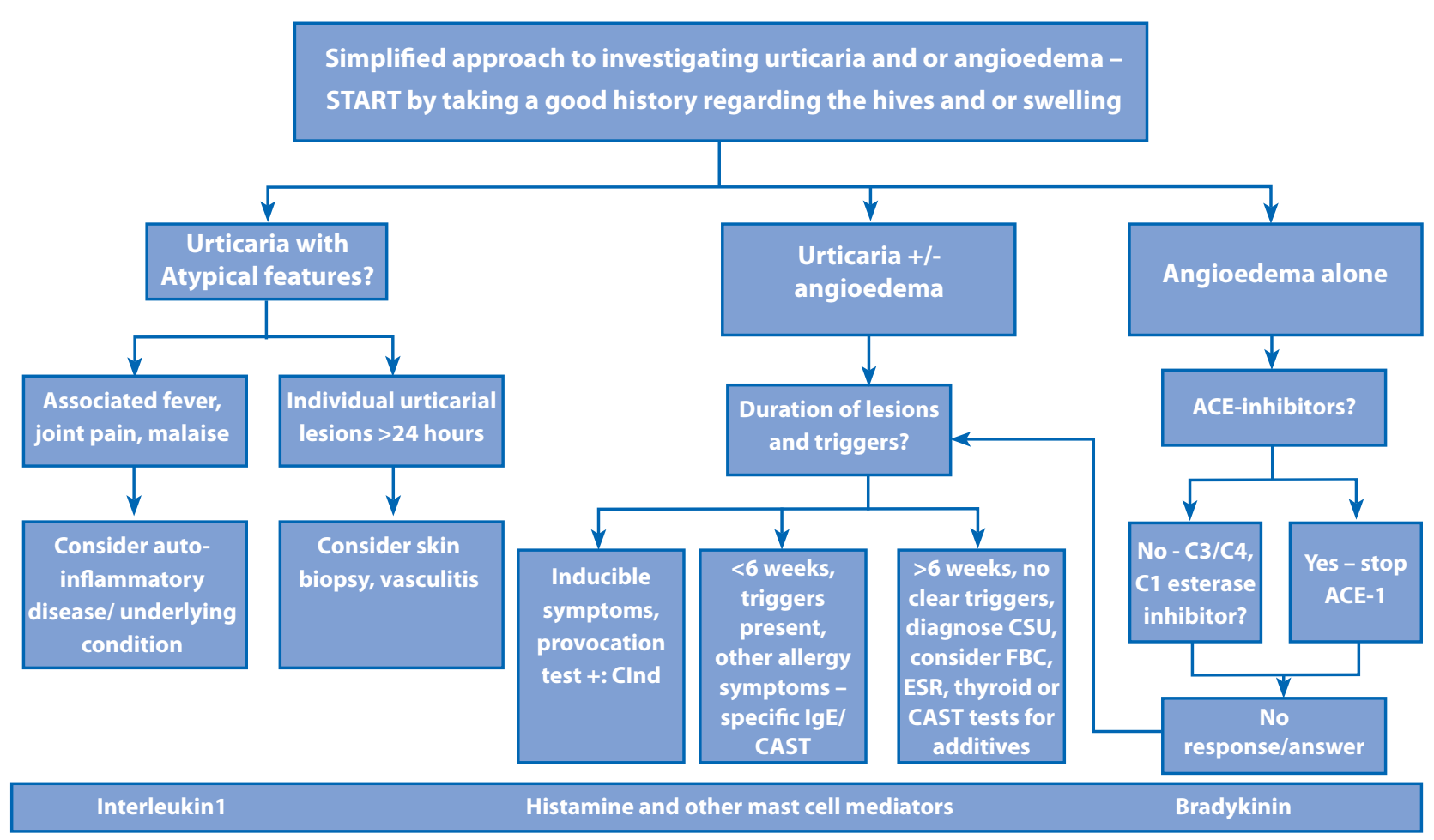

Figure 1. Approach to investigation of urticaria and angioedema, adapted by the author from EAACI/GA²LEN/EDF/WAO 2013 guideline diagnosis algorithm. ${ }^{4,11,16}$

auto-immune, thyroid and other medical conditions, as well as possible triggers such as stress, medications or food additives., 5,9

Urticarial vasculitis or auto-inflammatory conditions should be considered where lesions remain longer than 24 hours, is indurated and painful or systemic symptoms such as fever and arthralgia are present.

\section{Chronic Spontaneous Urticaria - History and clinical evaluation}

Enquiry should be made regarding the timing, onset and nature of hives, presence or absence of angioedema and possible triggering or perpetuating factors. Medical and family history, medications, travel, infections, surgery and dental implants, psychological conditions, hormonal and physical factors, relationship to food and atopy should be evaluated. A patient diary may be helpful in eliciting a pattern. History or findings suggestive of auto-immunity, thyroid disease and haematological diseases demand attention. ${ }^{4}$

\section{Trigger factors}

Drugs, especially aspirin and non-steroidal anti-inflammatory medications, should be withdrawn if suspected..$^{13}$ Excipients should be considered when evaluating adverse drug reactions, especially when a patient has previously tolerated a different manufacturer's version of the same drug. Excipients are not always listed in the MIMS or package inserts, but can be obtained directly from the manufacturers or with the aid of the UCT Department of Pharmacology's Medicine Information Centre.

CSU may be also be aggravated by dose-dependent delayed hypersensitivity reactions presenting 4-12 hours after ingestion of food components, such as preservatives, colourants and taste intensifiers. Benzoate derivatives (E210-219) are commonly implicated and ubiquitously added to cool drinks, condiments, sauces and medication formulations as anti-oxidant. Nitrates in processed meat products, monosodium glutamate and tartrazine are other common examples. Naturally occurring substances such as glutamate, histamine and salicylates may also be implicated. These 'pseudo-allergen' intolerances are episodic and dose dependent, and co-factors such as alcohol or exercise may be involved. Reactions may occur upon cumulative exposure - the 'kiddies' party' or 'pizza restaurant' effect! Published responder rates for elimination diets low in pseudoallergens vary in the range of $30-90 \%$, with $20-60 \%$ following challenge testing. ${ }^{10}$

Current EAACI guidelines allow for a trial of elimination diet in persistent cases. $^{16}$ Cellular Antigen Stimulation Tests (CAST) or basophil activation assays can also be helpful. ${ }^{13}$ Infections such as occult dental abscesses, Helicobacter pylori and parasitic infections should be treated if present.

\section{Associations with auto-immunity, underlying medical conditions and psychological factors}

There is an association between CSU and auto-immunity, with autoreactive antibodies triggering the high affinity mast cell receptors directly in estimated $30-40 \%$ of CSU cases. The Autologous Serum Skin Test (ASST) can be used to demonstrate auto-reactivity to the patient's own serum, eliciting a wheal and flare reaction upon intradermal injection. ${ }^{9,13}$ Basophil upregulating activity (increased DC63 or CD 203) has been found after incubation with patients' own sera. CSU is often associated with elevated anti-thyroid peroxidase and antithyroglobulin antibodies, even in presence of normal thyroid function. Thyroxine treatment in euthyroid patients with 
elevated antibodies is not currently recommended, although there are reports of benefit in some cases. These patients should be advised to have their thyroid function evaluated regularly. ${ }^{6,8}$ CSU associated with thyroid disease or auto-immunity tend to be more severe and persistent. Neoplastic and haematological conditions such as lymphoma and mastocytosis may occasionally present as urticaria. ${ }^{3,12}$

Psychological factors are important as triggers or perpetuating factors of CSU. Symptoms tend to flare due to stress or important life events. Urticaria also causes distress due to the intense pruritus and embarrassing appearance, creating a vicious cycle. Depression, functional and sleep impairment are common and it is important for the practitioner to offer emotional support.

\section{Investigation}

The majority of cases need limited investigation, in order to exclude serious underlying disease. Extended investigation should be targeted and based on history and clinical evaluation.

Table 1: Recommended investigation of CSU according to EAACI/ GA²LEN/EDF/WAO 2013 guideline ${ }^{16}$

\begin{tabular}{ll}
\hline Routine & Extended \\
\hline Differential blood count & Infectious diseases \\
ESR, CRP & lgE mediated allergy \\
\hline Omission of suspected drugs & Skin tests \\
& Auto-antibodies \\
& Thyroid hormones \\
& Pseudo allergens \\
& Serum tryptase \\
& ASST (auto-reactivity) \\
& Skin biopsy (vasculitis) \\
\hline
\end{tabular}

\section{Non Pharmacological management}

Patients should reduce triggers, e.g. heat, stress and alcohol, and avoid medications and additives that may be implicated. Blanket avoidance of allergenic foods such as peanuts, egg, dairy or shellfish is unhelpful unless specific allergies have been demonstrated. Physical stimuli such as rubbing, scratching or pressure should be avoided and cooling lotions may be helpful. It is important to develop a partnership with the patient, taking time to explain the pathogenesis and create realistic expectations. Most patients are better within a year, some continue to be symptomatic for several years and a minority follows a long-term severe and relapsing course..$^{4,14}$

\section{Pharmacological Management}

Guidelines recommend second generation non-sedating antihistamines, e.g. cetirizine, levocetirizine, loratidine, desloratidine, fexofenadine or rupatidine, as first line therapy. ${ }^{16}$ If there is no response, the prescribed dose may be increased up to 4 times, as recommended second line therapy. ${ }^{2,16}$ Individual response varies so agents from different chemical classes could be tried. Older antihistamines should be avoided due to sedative and anticholinergic side effects.
Third line treatment includes montelukast, omalizumab and cyclosporine. Montelukast may be helpful in aspirin sensitive patients where the leukotriene pathway may be involved. Cyclosporine is an immunosuppressive medication that has been used successfully "off label" in refractory cases and remains included in international guidelines. Blood pressure, renal function and therapeutic drug levels should be monitored.

Omalizumab is a biological anti-lgE antibody indicated for severe allergic asthma and adult and adolescent CSU patients unresponsive to antihistamines. ${ }^{1}$ It is administered monthly via subcutaneous injection, in a hospital setting, due to a small risk of anaphylaxis. Omalizumab binds to the C-epsilon-3 domain of human IgE, thereby blocking the binding of IgE to the Fc-epsilon receptor of mast cells and basophils. ${ }^{15}$ In response the mast cells seem to downregulate high affinity lgE receptors and become less responsive. It is effective in approximately $75 \%$ of cases, but cost and accessibility are major barriers. 1,2,9,15

Short courses of prednisone up to 10 days can be useful for exacerbations, but long term reliance on oral or parenteral corticosteroids, including antihistamine/steroid combination tablets, should be avoided due to unacceptable steroidogenic side-effects, such as weight gain and dysregulation of blood glucose and bone density. ${ }^{16}$

$\mathrm{H} 2$ receptor blockers (cimetidine, ranitidine) are alternative agents but there is limited evidence for their efficacy. Ketotifen has antihistamine and mast cell stabilizing properties, but is locally only available in a syrup formulation. Evidence is limited but seems to support its efficacy, however there is some potential for sedation. Other options include dapsone, methotrexate and dosulpin.

\section{Conclusion}

Taking a good history is key when evaluating a patient with urticaria. Targeted investigations, withdrawal of suspected medications and pseudo-allergens, symptom control with nonsedating antihistamines and emotional support are the main pillars of management. Stress, physical factors, drugs and food additives are potentially modifiable trigger factors in chronic urticaria.

Refractory cases should be considered for biological or immunosuppressive therapy. These conditions need to be taken seriously as they have a severe impact on quality of life and may be associated with underlying medical disorders. There is a renewed interest in urticaria, with the third Global Urticaria Forum in November 2016. The idea is to increase global cooperation and the standard of care, by updating guidelines, creating a Chronic Urticaria Registry and Urticaria Centers of Reference and Excellence. ${ }^{15}$ In South Africa, Allergology Fellows and Diplomats, immunologists and dermatologists may be able to assist with complex or non-responsive cases.

\section{Acknowledgements}

The author wishes to thank Prof PC Potter for his teaching, guidance and mentorship in the practice of Allergology, as well as for proofreading and input regarding this manuscript. There is no conflict of interest to declare. 


\section{References}

1. Casale TB. Omalizumab for chronic urticaria. J Allergy Clin Immunol Pract. 2014;2(1):118-9.

2. Casale TB, Bernstein JA, Maurer M, et al. Similar Efficacy with Omalizumab in Chronic Idiopathic/Spontaneous Urticaria Despite Different Background Therapy. J Allergy Clin Immunol Pract 2015; 3(5):743-50.

3. Chen $Y$, Wu C, Shen J, et al. Cancer Risk in Patients with Chronic Urticaria: A Population-Based Cohort Study. Arch Dermatol. 2012;148(1):103-8.

4. Emanuel S, Hawarden D. Urticaria and angio-edema. Curr Allergy Clin Immunol. 2013;26(1):31-5.

5. Kaplan A. Therapy of chronic urticaria: a simple, modern approach. Ann Allergy Asthma Immunol. 2014;112:419-25.

6. Kim DH, Sung NH, Lee AY. Effect of Levothyroxine Treatment on Clinical Symptoms in Hypothyroid Patients with Chronic Urticaria and Thyroid Autoimmunity. Ann Dermatol. 2016;28(2):199-204.

7. Lindelof $B$, Sigurgeirsson $B$, Wahlgren $C F$, et al. Chronic urticaria and cancer: an epidemiological study of 1155 patients. Br J Dermatol. 1990;123:453-6.

8. Lunge $S B$, Borkar $M$, Pande $S$. Correlation of serum antithyroid microsomal antibody and autologous serum skin test in patients with chronic idiopathic urticaria. Indian Dermatol Online J. 2015;6(4):248-52.
9. Makris MP, Papadavid E, Zuberbier T. The use of biologicals in cutaneous allergies - present and future. Curr Opin Allergy Clin Immunology. 2014;14(5):409-16.

10. Maurer M, Grattan CE, Zuraw L. Urticaria and angioedema without wheals. 2012 in Holgate S ed. Allergy. 4th Edition, Elsevier Saunders, Chapter 12, p. 247-61.

11. Maurer M, Church M K, Marsland G, Sussman F, Siebenhaar C, Vestergaard C, Broom, B. Questions and answers in chronic urticaria: where do we stand and where do we go? Chronic Urticaria, Challenges, Insights and New Directions, Proceedings of the second Global Urticaria Forum, November 2015, Berlin. JEADV 2016;30(Suppl. 5):7-15.

12. Mohamed Z. Urticaria as Manifestation of Hodgkin's lymphoma. Indian J Allergy Asthma Immunol 2015;29:88-91.

13. Motala C. Investigation and management of chronic urticaria. CME. September 2003;3(9):430-8.

14. Muller B. Urticaria and Angioedema: A Practical Approach. Am Fam Physician 2004;69(5):1123-8.

15. Ring J. Chronic Urticaria: new hope for an old disease. Chronic Urticaria, Challenges, Insights and New Directions, Proceedings of the second Global Urticaria Forum, November 2015, Berlin. JEADV 2016;30(Suppl. 5):3-4.

16. Zuberbier T, Aberer W, Asero R, et al. The EAACI/GA2LEN/EDF/WAO Guideline for the definition, classification, diagnosis, and management of urticaria: the 2013 revision and update. Allergy 2014;69(7):86887. 